\title{
Prediction of Ultrasonic Parameters of Mortar by using Artificial Neural Networks Techniques
}

\author{
Abdelilah Dariouchy \\ LMTI, Faculte Des Sciences, \\ Universite Ibn Zohr, Agadir, Maroc
}

\author{
Hicham Lotfi \\ LMTI, FSA-Agadir, Maroc \\ EST Laayoune, Université Ibn Zohr, Maroc
}

\begin{abstract}
Two artificial neural networks (ANN) models are developed to predict the evolution of group velocity and peak to peak amplitude of an ultrasonic wave propagate in mortar, also we these models use it to know the acoustic impedance during hydration of mortar specimen. The useful data bases to train and to test the performances of the models are collected from the experiences done on the mortar dough.

In this study, the temperature, the mass reports of cement on sand, water on cement and the time of the manipulation, are retained like relevant entries of our models. Several network configurations are evaluated. For the two architectures of models, the optimal model selected is an ANN with only one hidden layer. These models are able to predict respectively group velocity, acoustic impedance and peak to peak amplitude evolution with the means relative errors (MRE) of $0.29 \%$ and $0.35 \%$ and $4 \%$.
\end{abstract}

\section{Keywords}

Group velocity; Acoustic impedance; Peak to peak amplitude; Neural network; Back-propagation algorithm

\section{INTRODUCTION}

The mortar is a heterogeneous composite material; it is composed of cement Portland, sand and water [1]. In order to control the quality of the civil genius materials, an acoustic study is made to investigate the proprieties of mortar specimen. At the time of the experiences done, several parameters are taken into account such as the temperature T, the mass reports of cement on sand $\mathrm{Mc} / \mathrm{Ms}$ and water on cement $\mathrm{Me} / \mathrm{Mc}$. The variation of these parameters makes it possible to know the evolution of the mortar in function with hydration time.

In a container consisted by Plexiglas and glass enclosing the mortar sample, this one is immersed in a water tank and thermostated. The container is excited by a transducer of central frequency $0.5 \mathrm{MHZ}$, echoes appear with the interface Plexiglas/mortar and mortar/glass. The treatment of signals backscattered by permit to determinate the rigidity of mortar. From the acoustic parameters of this one, it is possible to determine indirect way the physical and chemical properties of the mortar. Starting from these echoes, it is easy to go up at the group velocity $\mathrm{Cg}$ and acoustic impedance $\mathrm{Z}$ [2]. However, in order to be able to realize one of these experiments, three days should at least be waited, so that the hardening of cement is completed, which is long in terms of time, then the idea to use the techniques of the artificial neural networks to predict for different $\mathrm{T}$ and various mass reports, characteristics of the mortar.

In this study, the group velocity $\mathrm{Cg}$, the acoustic impedance $\mathrm{Z}$ and the peak to peak amplitude backscattered by the mortar, are selected like relevant parameters characterizing the mortar. The prediction of the experiment evolution by ANN will enable us to play on the speed of hardening in order to ensure a gain of time and economy. An ANN is only neurons organized in layers, which have a very great capacity of calculation [3]. The latter are a statistical tool for analysis making it possible to build a model of behavior starting from a certain number of examples. In this work, two models of ANN with four entries, two outs and one out are developed. These last are able to predict the group velocity, the acoustic impedance and peak to peak amplitude for various temperatures $\mathrm{T}$ and various reports of $\mathrm{Mc} / \mathrm{Ms}$ and of $\mathrm{Me} / \mathrm{Mc}$ on an interval of three days.

\section{SIGNALS BACKSCATTERED BY MORTAR}

The mortar is a heterogeneous material obtained by mixing cement Portland, sand and water. In this study, all mortars specimen prepared with Cement CPJ 45 produced by factory of Agadir, Morocco. Sand used is extracted from the Oued Souss River. The water used for hydration is the water of the city of Agadir.

The attempts realized in the laboratory are made in normal Reflection [4]. After the preparation and mixing the mortar sample this one is putting in a parallelepiped container, the last one is immersed in a water tank and thermostated. Knowing that the parallel faces of the container are constituted by Plexiglas plates of thickness $d=20 \mathrm{~mm}$, and glass of thickness $D_{v}=3 \mathrm{~mm}$, the thickness of the mortar enclosed is $\mathrm{L}=15 \mathrm{~mm}$. The container immersed is excited in its perpendicular plan by a transducer of central frequency 0.5 MHZ.

The incident signal follows the path presented in the Figure 1. $A_{1}, A_{2}$ and $A_{3}$, are the different echoes backscattered by the container contains the mortar sample, $\mathrm{A}_{0}$ is the incident signal. Figure 2 represents a backscattered signal by a container enclosed mortar. This signal is composed of three echoes:

- Echo $\mathrm{A}_{1}$ corresponds to reflection of the incident wave with the interface between water and the first face Plexiglas.

- Echo $\mathrm{A}_{2}$ corresponds to the reflection of interface between the second Plexiglas face and the mortar enclosed.

- Echo $\mathrm{A}_{3}$ corresponds to the reflection of interface between the mortar and the first face of glass plate. 


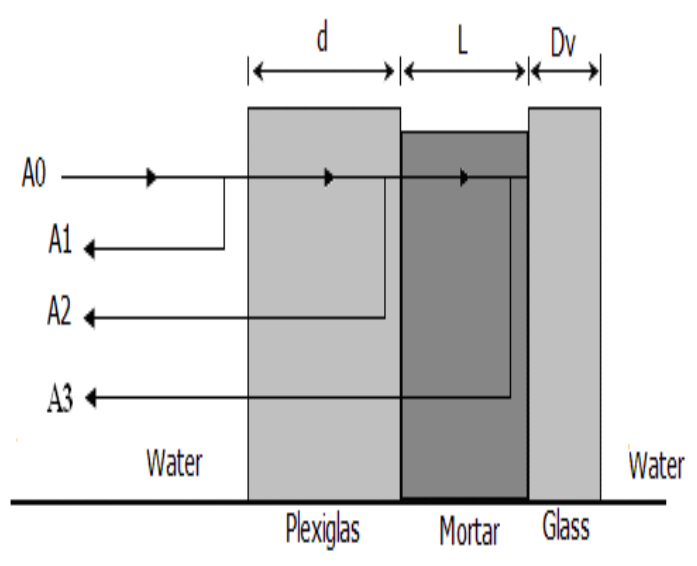

Fig 1: Mechanisms of formation of echoes $A_{1}, A_{2}$,

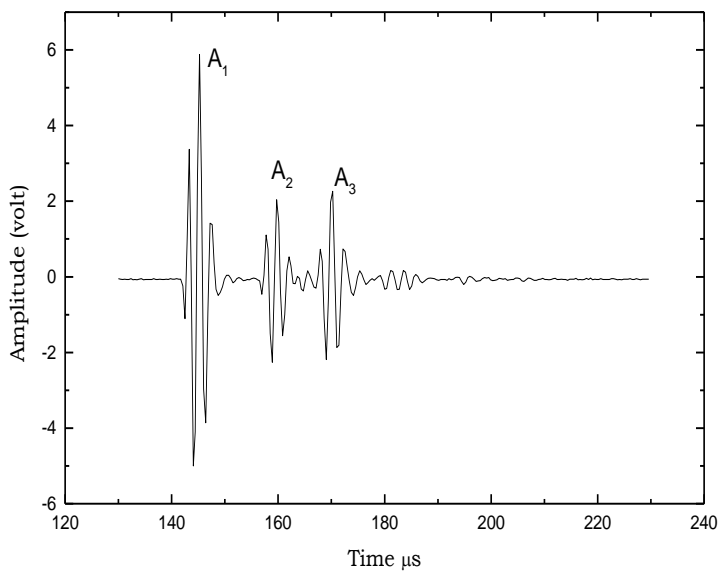

Fig 2: Signal backscattered by a container enclosed the mortar

\section{METHODOLOGY}

The development of a neural network model requires a data set of training and testing. Indeed, the neural networks fulfil parameterized nonlinear functions. They can approach any function of regression if a model which makes minimum of the cost function of least squares exists. Also, the implementation of a neural network requires [3]:

- A collection of the necessary data for the training and the validation;

- A training of the neural network model, and the evaluation of the configuration which leads to the selection of the optimal model;

- A validation of the optimal model with test data which are not used in the training phase;

Artificial Neural Network (ANN) architecture comprises mainly parallel adaptive processing elements with hierarchical structured interconnected networks. Each processing unit of an ANN has multiple inputs and a single output. The relationship between the input and the output signals are usually formulated as follows [7]:

$$
\begin{aligned}
& O_{j}=f\left(y_{j}\right)=\frac{1}{1+\exp \left(y_{j}\right)} \\
& y_{j}=\sum_{i=1}^{n} w_{j i} I_{i}-\theta_{i}
\end{aligned}
$$

Where $\mathrm{Oj}$ is the output signal of the jth unit, yj the potential of the $\mathrm{jth}$ unit, $\mathrm{f}$ the activation function that is a sigmoidal function in this case, wji the connection weights between ith and jth units, $\theta i$ the threshold value of the ith unit and $n$ the number of input signals. The figure 3 shows a three-layer neural network. All the units are formed into a multiple layers, i.e. an input layer, a hidden layer and an output layer. The basic idea of training a neural network is as follows. First, the square error of the pth training pattern Ep is defined as:

$$
E_{p}=\frac{1}{2} \sum_{k=1}^{m}\left(T_{p k}-O_{p k}\right)^{2}
$$

Where Tpk is the teacher signal (desired output) to the kth output unit for pth training pattern, Opk is the output signal to the kth output unit for pth training pattern and $m$ the number of output units. In the training process, wji and $\theta \mathrm{i}$ are modified repeatedly based on gradient descent method to minimize the above error. This modification proceeds downward. Through such an iterative process, the network attains promptly the ability similar signal to the teacher's one. This training algorithm is called back-propagation neural network [3].

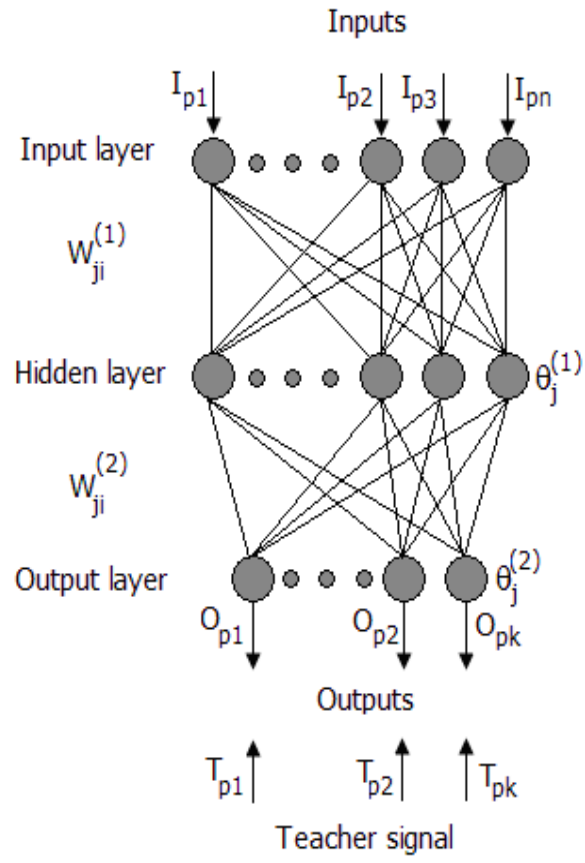

Fig 3: Layout of three layer back propagation neural network

\subsection{Collection of data}

The conception of the neural network model requires the determination of the relevant entries which have a significant influence on the required model. In this work, a data base is collected to train and to test the performance of two ANN models. The temperature $\mathrm{T}$, the mass reports of cement on sand $\mathrm{Mc} / \mathrm{Ms}$, the water on cement $\mathrm{Me} / \mathrm{Mc}$, and the time of the manipulation, are retained like relevant entries of our models. The collected data for the training and the validation phases of the neural network model are represented in Table 1. 
Table 1. Data set collected for training of two ANN models

\begin{tabular}{|c|c|c|c|c|}
\hline $\mathbf{T}\left({ }^{\circ} \mathbf{C}\right)$ & $\mathbf{4 2}$ & $\mathbf{3 7}$ & $\mathbf{3 5}$ & $\mathbf{4 2}$ \\
\hline $\begin{array}{c}\text { Report } \\
\text { Mc/Ms }\end{array}$ & 0.50 & 0.50 & 0.50 & 0.40 \\
\hline $\begin{array}{c}\text { Report } \\
\mathrm{Me} / \mathrm{Mc}\end{array}$ & 0.650 & 0.800 & 0.500 & 1.575 \\
\hline Time (h) & $(15-72.5)$ & $(15-72.5)$ & $(15-72.5)$ & $(15-72.5)$ \\
\hline $\begin{array}{c}\text { Velocity Cg } \\
\text { (m/s) }\end{array}$ & $\begin{array}{c}(2123- \\
2799)\end{array}$ & $\begin{array}{c}(1853- \\
2503)\end{array}$ & $\begin{array}{c}(2060- \\
2883)\end{array}$ & $\begin{array}{c}(1757- \\
2405)\end{array}$ \\
\hline $\begin{array}{c}\text { Impedance Z } \\
(106 . N . s / m 3)\end{array}$ & $\begin{array}{c}(3.97- \\
7.67)\end{array}$ & $\begin{array}{c}(3.60- \\
4.80)\end{array}$ & $\begin{array}{c}(5.02- \\
6.30)\end{array}$ & $(3.50-4.60)$ \\
\hline $\begin{array}{c}\text { Amplitude } \\
\text { (volt) }\end{array}$ & $\begin{array}{c}(16.71- \\
6.45)\end{array}$ & $\begin{array}{c}(14.81- \\
3.74)\end{array}$ & $\begin{array}{c}(11.75- \\
5.72)\end{array}$ & $\begin{array}{c}(14.02- \\
3.73)\end{array}$ \\
\hline
\end{tabular}

\section{Training of the model}

Using the whole of the characteristics of the tubes as training and validation bases, several models are learned. But before all things, it is indispensable to normalize all variables of entry. Indeed, if some entries have very different sizes, those that are small don't have an influence on the training. Once the data is normalized, it remains to determine the artificial neural network architecture. In our work, the perception multi-layers (PML) is used as model of network architecture employed to predict the evolution of group velocity, acoustic impedance and peak to peak amplitude of mortar. This last parameter has a directed network of artificial neurons organized in layers and where information travels only one direction of the layer to entry towards the output layer. The layer of entry always presents a virtual layer associated with the entries of system; it does not contain any neuron. The layers which follow the latter are layers of neurons, where the neurons are connected between them by the ponder connections. They are the weights of these connections which control the operation of network [3].

Indeed, the number of neurons in each hidden layer can vary according to the complexity of the problem and the whole of data. In this work, the number of hidden layer was varied from 1 to 2, the architecture of this network is represented in Figure 4.
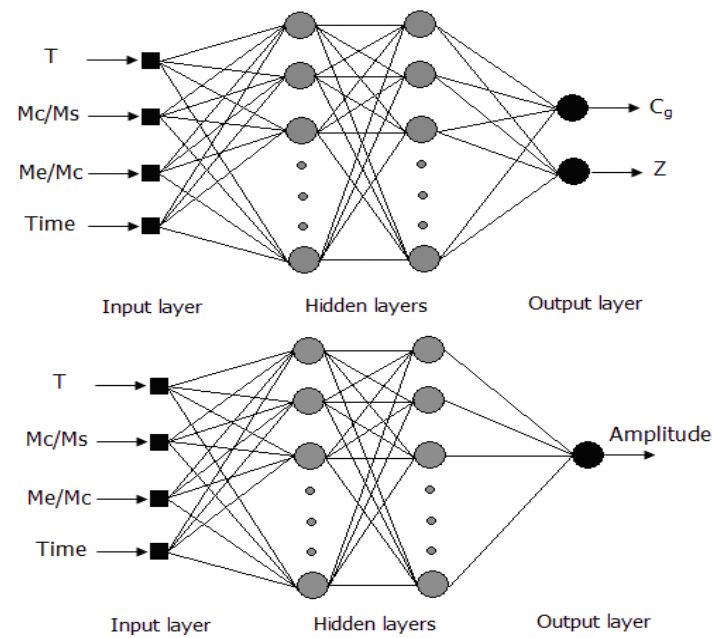

Fig 4: Architectures of the two models of ANN to predict the evolution of the $\mathrm{Cg}, \mathrm{Z}$ and the peak to peak amplitude of mortar

The optimal configuration is based on the minimization of the difference between the desired and the predicted output by the ANN model. The back-propagation algorithm of the gradient which uses the technique of supervised training is employed to learn the hidden layers of the model of the artificial neural network. The sigmoid function is regarded as transfer function of the neurons which undergo sharp variations when the values of the parameters are high for the entries of the hidden neurons. Indeed, not to leave the linear part of sigmoid, the synaptic weights are initialized between -0.5 and +0.5 .

\section{SELECTION OF THE OPTIMAL MODEL}

The biais - variance dilemma permits to find a model which carries out the best compromise between the training and the generalization capacities [5]. The selection of models is done comparing the errors of each artificial neural network configuration, calculating the mean relative error (MRE), the mean absolute error (MAE) and the standard error (SE) of the predicted output of network [6]. The coefficients of correlation $\mathrm{R}$ is used like performance measures of the model between the predicted and the desired outputs. The three errors measures and the coefficient of correlation are given by the following relations:

$$
\begin{array}{r}
\text { MAE }=\frac{1}{N} \sum_{i=1}^{N}\left|C_{i}-O_{i}\right| \\
M R E=\frac{1}{N} \sum_{i=1}^{N} \frac{\left|C_{i}-O_{i}\right|}{C_{i}} \\
S E=\frac{\sqrt{\sum_{i=1}^{N}\left(C_{i}-O_{i}\right)^{2}}}{N-1} \\
R=1-\frac{\sum_{i=1}^{N}\left(C_{i}-O_{i}\right)^{2}}{\sum_{i=1}^{N}\left(C_{i}-O_{m}\right)^{2}}
\end{array}
$$

Where $\mathrm{N}$ is the number of data, $\mathrm{Ci}$ and $\mathrm{Oi}$ are the desired and the predicted values of the model respectively. Om is the mean of the predicted values.

\section{RESULTS AND DISCUSSION}

Once the learned network, its performance is evaluated initially by the same unit used in the training phase, then by the reserved set for the validation phase. The analysis is repeated several times. Indeed, the values of errors are measured for each ANN architecture. The results of the predictions values are presented in Table 2 for one hidden layer.

Table 2. Parameters errors of prediction of group velocity, acoustic impedance and amplitude, with different ANN configurations.

\begin{tabular}{|c|c|c|c|c|c|}
\hline $\begin{array}{c}\text { Output of } \\
\text { ANN }\end{array}$ & $\begin{array}{c}\text { Nbr of } \\
\text { neurons } \\
\text { by layer }\end{array}$ & MAE & MRE & SE & $\mathbf{R}^{\mathbf{2}}$ \\
\hline \multirow{2}{*}{$\begin{array}{c}\text { Velocity } \\
\text { C }_{\mathrm{g}} \text { (m/s) }\end{array}$} & 4 & 0.0035 & $0.90 \%$ & 0.00073 & 0.9989 \\
\cline { 2 - 6 } & 6 & 0.0025 & $0.63 \%$ & 0.00051 & 0.9994 \\
\hline $\left.\begin{array}{c}\text { impedance } \\
\text { Z } \\
\left(10^{6} . \text { N.s/m }\right.\end{array}{ }^{3}\right)$ & 8 & 0.0011 & $0.29 \%$ & 0.00026 & 0.9998 \\
\cline { 2 - 6 } & 6 & 0.0042 & $1.24 \%$ & 0.00089 & 0.9988 \\
\hline $\begin{array}{c}\text { amplitude } \\
\text { (volt) }\end{array}$ & 8 & 0.0018 & $0.55 \%$ & 0.00045 & 0.9996 \\
\cline { 2 - 6 } & 6 & 0.0015 & $0.35 \%$ & 0.00041 & 0.9997 \\
\cline { 2 - 6 } & 8 & 0.0097 & $4.99 \%$ & 0.00170 & 0.9810 \\
\hline \multirow{2}{*}{} & & 0.0077 & $3.94 \%$ & 0.00156 & 0.9840 \\
\hline
\end{tabular}


For the first model, the best configuration is found for an ANN model with only eight neurons in the hidden layer. The MRE, MAE and SE for this optimal model are respectively $0.29 \%, 0.0011(\mathrm{~m} / \mathrm{s})$ and $0.00026(\mathrm{~m} / \mathrm{s})$ for the first output $\mathrm{Cg}$. On the second output $\mathrm{Z}$ the values of these errors are $0.35 \%$, 0.0015 (106.N.s/m3) and 0.00041 (106.N.s/m3) respectively. For the second model, the best configuration is found for an ANN model with only six neurons in the hidden layer. The MRE, MAE and SE for this optimal model are 3.94\%, 0.0077 (volt) and 0.00156 (volt) respectively. To reveal the credibility of prediction from optimal configurations, the predicted values are traced according to the desired values. The results obtained show the good agreement between the predicted values and the desired values. The coefficients of determination $\mathrm{R}^{2}$ for our optimal models are 0.9998 for group velocity $\mathrm{C}_{\mathrm{g}}$ (Figure 5), 0.9997 for acoustic impedance $\mathrm{Z}$ (Figure 6) and 0.984 for amplitude (Figure 7).

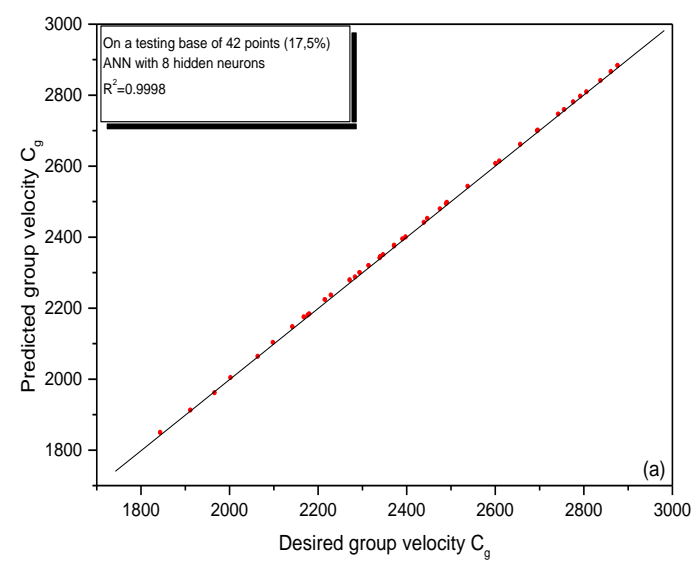

Fig 5: Correlation of desired versus neural network values of the group velocity

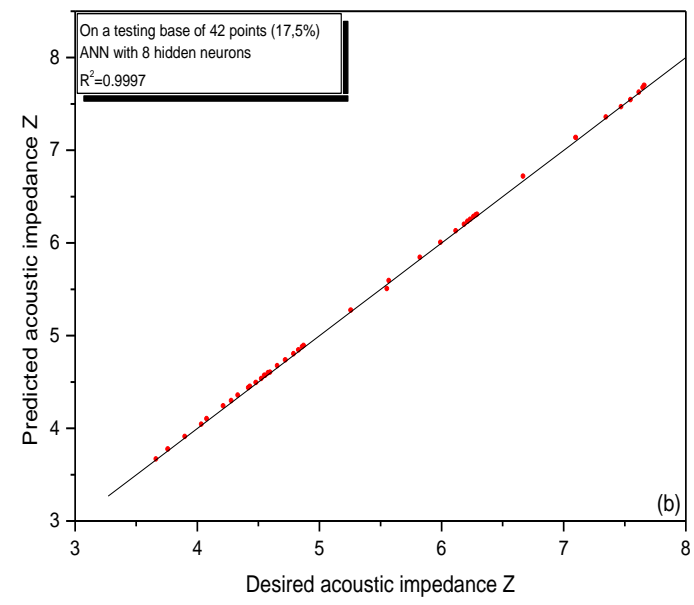

Fig 6:Correlation of desired versus neural network values of the acoustic impedance.

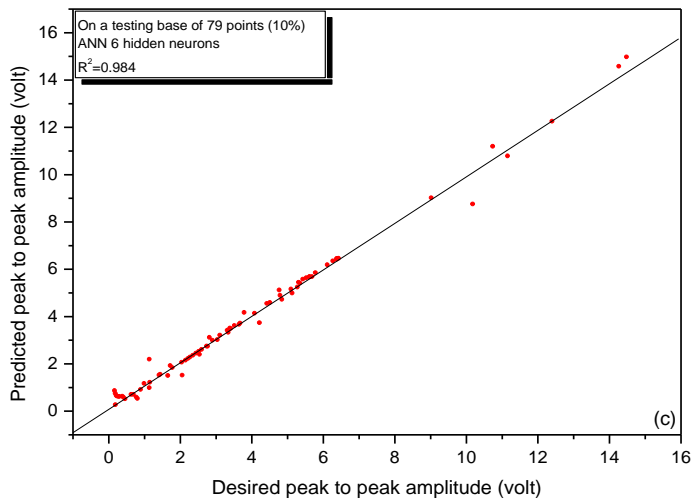

Fig 7: Correlation of desired versus neural network values of the peak to peak amplitude.

The figures 8 and 9 show the group velocity and the acoustic impedance evolution for different $\mathrm{T}$ and various mass reports. This model is applied successfully to predict the $\mathrm{Cg}$ and $\mathrm{Z}$ evolution, for $\mathrm{T}=35^{\circ} \mathrm{C}, \mathrm{Mc} / \mathrm{Ms}=0.5$ and $\mathrm{Me} / \mathrm{Mc}=0.6$ and $\mathrm{Me} / \mathrm{Mc}=0.7$. The figure 7 shows the peak to peak amplitude evolution predicted for the $\mathrm{T}=35^{\circ} \mathrm{C}, \mathrm{Mc} / \mathrm{Ms}=0.5$ and $\mathrm{Me} / \mathrm{Mc}=0.6$ and $\mathrm{Me} / \mathrm{Mc}=0.7$.

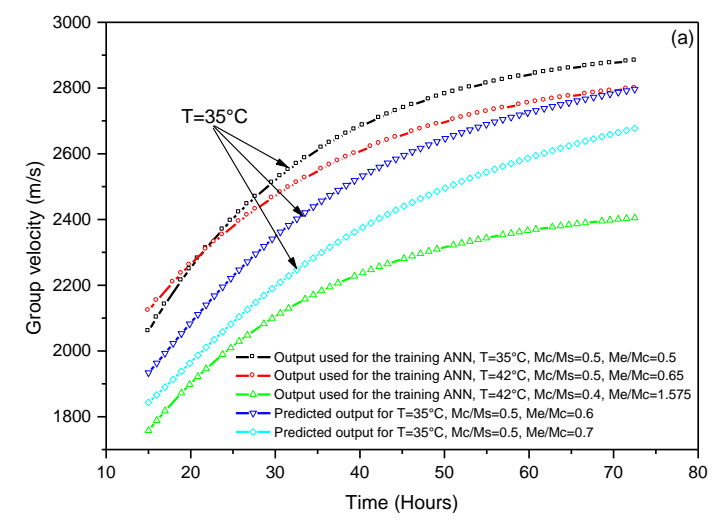

Fig 6: Prediction of the group velocity for $\mathrm{T}=35^{\circ} \mathrm{C}$ with different mass reports.

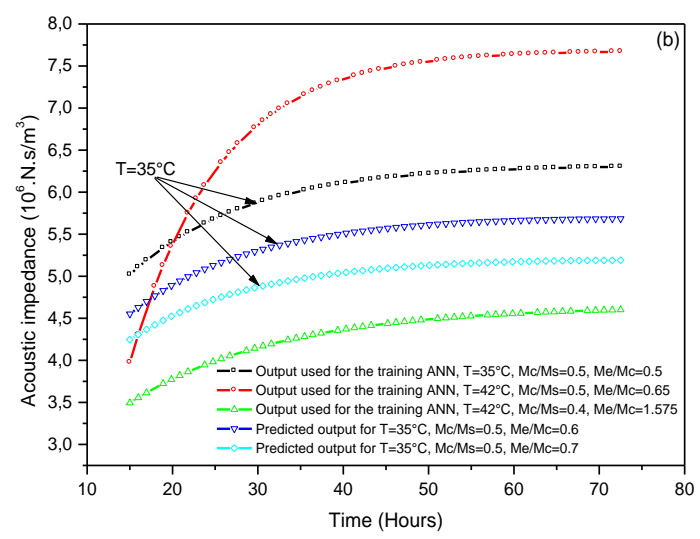

Fig 9: Prediction of the acoustic impedance for $\mathrm{T}=35^{\circ} \mathrm{C}$ with different mass reports. 
The figure 10 shows that the curves nearly have the same pace, however they different by the time of the hold (minimal amplitude). From the results predicted for the temperature $\mathrm{T}=35^{\circ} \mathrm{C}$ and a report $\mathrm{Mc} / \mathrm{Ms}=0.5$ fixed, the time of the hold increases with the increase of the report mass $\mathrm{Me} / \mathrm{Mc}$. This figure shows the different periods of hydration of the mortar $[8,9]$ :

(1): the initial period, (2): the dormant period,

(3): the hold period, (4): the hardening period.

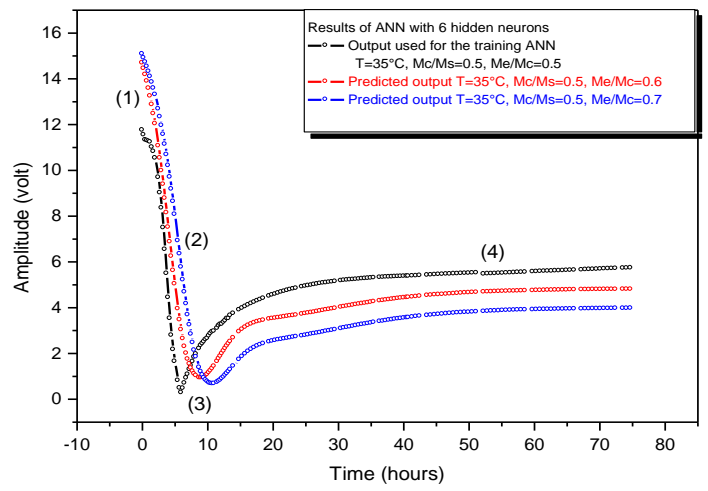

Fig 10: Prediction of the peak to peak amplitude evolution for $\mathrm{T}=35^{\circ} \mathrm{C}$ with different mass reports.

The calculation of the mean quadratic errors of training EA and testing ET during the learning phase, made it possible to visualize the network training evolution and also to see which iteration training must be stopped. The expression of the two errors is given by the following relations [7], Where NA and NT are the numbers of training and testing data respectively.

$$
\begin{gathered}
E_{A}=\sqrt{\frac{\sum_{i=1}^{N_{A}}\left(y_{i}-y_{d i}\right)^{2}}{N_{A}}} \\
E_{T}=\sqrt{\frac{\sum_{i=1}^{N_{T}}\left(y_{i}-y_{d i}\right)^{2}}{N_{T}}}
\end{gathered}
$$

The figure 11 shows the evolution of the mean quadratic errors at the time of the training in the case of the prediction of the peaks to peaks amplitudes values.

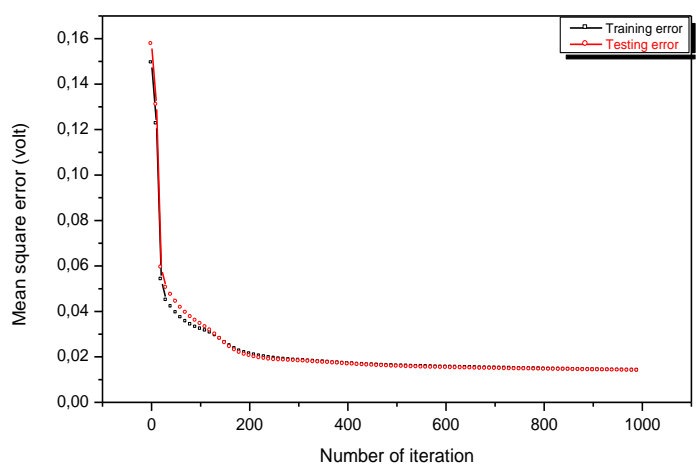

Fig 11: Training and testing error in function number of iterations

\section{CONCLUSION}

In this work, two ANN models are developed, in order to predict the evolution of tree ultrasonic parameter in function of time de hydration. The optimal model for these two types of ANN involve only one hidden layer, with eight neurons for the first and six neurons for the second model. These last are able to predict respectively the group velocity, the acoustic impedances and the peak to peak amplitudes values for different temperatures and different mass reports with an error MRE of $0.29 \%, 0.35 \%$ and $3.94 \%$. The neural networks techniques permit also to show the evolution of the hardening phase during time of hydration. From the predicted results, we observed the increase of time of hydration of the mortar, and then depend to the temperature and the mass report of water on cement.

Therefore, this configuration of ANN constitutes an appreciable help to predict the hardening speed and to ensure a considerable profit of time and materials.

\section{REFERENCES}

[1] Aggelis DG, Polyzos D, Philippidis TP. Wave dispersion and attenuation in fresh mortar: theorical predictions vs. experimental results. Journal of the Mechanics and Physics of Solids 2005; (53) 857-883.

[2] Philippidis TP, Aggelis DG. Experimental study of wave dispersion and attenuation in concrete. J. Ultrasonics (2004), Elseiver.

[3] Dreyfus G, Martinez JM, Samuelides M, Gordon MB, Badran F, Thiria S, Hérault L. Réseaux de neurones méthodologie et applications. Livre 2ème édition, 2004; (ISBN : 2-212-11464-8).

[4] H.Lotfi, B.Faiz, A.Moudden, D.Izbaim, A.Menou, G.Maze Et D.Decultot "Ultrasonic Characterization and Hardening of Mortar Using the Reflection Technique" ,journal High Temperature and Process, 2009.

[5] Geman S, Bienenstock E, Doursat R. Neural Networks and the bias/variance dilemma. Neural computation 1992; (4) pp. 1-58.

[6] Sablani SS, Baik OD, Marcotte M. Neural networks for predicting thermal conductivity of bakery products. Journal of food Engineering May 2002; (52) pp. 299304.

[7] Singh SK, Srinivasan K, Chakraborty D. Acoustic characterization and prediction of surface roughness. Journal of Materials Processing Technology March 2004; (152) pp. 127-130.

[8] REGOURD M. L'hydratation du ciment portland, le béton hydrolique. Paris presse de l'ENPC, sous direction de Jacques BARON et Raymond SAUTEREY, 1995; Chapitre 11, PP 191-211.

[9] HEWLETT C. Chemistry of cement and concrete, fourth edition. Edition by Peter LEA, 1998; pp 241-289. 